\title{
Growth of high-quality GaAs/AIAs Bragg mirrors on patterned InP-based quantum well mesa structures
}

\author{
H. Gebretsadik, K. Kamath, K. K. Linder, X. Zhang, and P. Bhattacharya \\ Department of Electrical Engineering and Computer Science, Solid State Electronics Laboratory, \\ University of Michigan, Ann Arbor, Michigan 48109-2122 \\ C. Caneau and R. Bhat \\ Bellcore, Redbank, New Jersey 07701-5699
}

(Received 2 May 1997; accepted for publication 30 May 1997)

\begin{abstract}
We have investigated the regrowth of GaAs/AlAs quarter-wave Bragg reflectors on patterned mesa InP-based quantum well heterostructures that can be fabricated into $1.55 \mu \mathrm{m}$ vertical cavity surface emitting lasers. It is seen from transmission electron and scanning electron microscopy that the multiple layer GaAs-based mirrors can be grown on InP-based heterostructure mesas of diameters $10-40 \mu \mathrm{m}$ without noticeable propagation of defects into the reflector layers or the quantum well region below. At the same time the photoluminescence from the quantum wells after regrowth indicates that lasers can be fabricated. (C) 1997 American Institute of Physics.
\end{abstract}

[S0003-6951(97)02231-6]

As with many other devices, the development of long wavelength $(\lambda=1.55 \mu \mathrm{m})$ InP-based vertical cavity surface emitting lasers (VCSELs) has been less rapid than the GaAsbased devices. Aside from the relative complexities in processing InP-based devices, there are three reasons for this that can be cited: Auger recombination and intervalence band absorption, less applicability of the selective wet oxidation of Al-bearing compounds, ${ }^{1}$ which has been so effective in $1 \mu \mathrm{m}$ VCSEL technology, ${ }^{2,3}$ and the difficulty in fabricating high-reflectivity semiconductor Bragg mirrors lattice matched to InP. It is the last factor which poses the most serious obstacle. Because of the small refractive index difference between InP and InGaAsP (or InGaAlAs) lattice matched to it, as many as 45 pairs of $\lambda / 4$ layers are required to get a high enough reflectivity. ${ }^{4}$ This presents both epitaxy and processing challenges. The two most prominent alternate mirror technologies that have emerged are high index contrast dielectric stacks ${ }^{5,6}$ and the wafer fusion technology. ${ }^{7}$ The former inherently gives rise to current injection problems due to the dielectric materials being poor conductors of heat and electricity. The wafer fusion technology in which GaAs/AlAs stacks are fused to both sides of the gain region do not pose any conductivity problems and looks most promising at the present time. However, the reliability of the technique and its application to full size wafers are yet to be ascertained.

It has been demonstrated that epitaxial growth on finite substrates can dramatically reduce the density of misfit dislocations present in the starting substrate ${ }^{8}$ or from the strain relaxation process once the critical thickness is reached during strained layer epitaxy. ${ }^{9}$ The reduction in dislocation density, or even complete elimination of misfit dislocations, occurs when growth is done on a patterned mesa surface or in a groove (trench). When the mesa size is reduced to the order of a few tens of microns, existing dislocations (possibly originating from the substrate) and surface inhomogeneities are greatly reduced. This inhibits the formation of misfit dislocations by the glide mechanism and the critical thickness is greatly enhanced. The small surface area will also inhibit dislocation multiplication by the Hagen-Strunk mechanism. ${ }^{10}$ If, in addition, sources of dislocation nucleation at the mesa edge can be minimized, the epitaxial overlayer on the mesa can be relatively defect-free and linear dislocation densities are less than $100 \mathrm{~cm}^{-1}$ along one of the $\langle 100\rangle$ directions. ${ }^{8}$ We have studied the photoresponse of strained $\operatorname{In}_{x} \mathrm{Ga}_{1-x}$ As $(0.05 \leqslant x \leqslant 0.20) \quad p-i-n$ photodiodes grown in 30-100 $\mu \mathrm{m}$ grooves of $1 \mu \mathrm{m}$ depth patterned in (001) GaAs substrates. ${ }^{11}$ The thicknesses of the diodes were $1 \mu \mathrm{m}$ and therefore much larger than the critical thickness. The diodes exhibited enhanced quantum efficiency and temporal response comparable to similar lattice matched photodiodes on planar substrates.

Since the lateral dimensions of low threshold current VCSELs vary from $2-10 \mu \mathrm{m}$ and the top distributed Bragg reflector (DBR) $\lambda / 4$ stack is of similar dimension, it is worthwhile to investigate the possibility of regrowing a GaAsbased DBR directly onto a patterned InP-based VCSEL wafer. In essence the InP-based VCSEL heterostructure would be grown up to the active region; and the wafer would be patterned into mesas. The top GaAs-based mirror heterostructure would be grown on it. The VCSEL would then be fabricated by standard photolithography and metallization techniques.

In the present study we have investigated the direct growth of GaAs/AlAs DBR mirrors on patterned mesas etched on InP-based multiquantum well (MQW) quantum well heterostructures, shown in Fig. 1. The first MQW structure (structure 1), shown in Fig. 1(a), was grown by molecular beam epitaxy (MBE) on (001) semi-insulating InP substrate, at a substrate temperature of $500{ }^{\circ} \mathrm{C}$. The structure has four $100 \AA \mathrm{In}_{0.53} \mathrm{Ga}_{0.47} \mathrm{As}$ wells separated by $100 \AA$ $\mathrm{In}_{0.52} \mathrm{Al}_{0.48} \mathrm{As}$ barriers and is similar to the active region of a $1.55 \mu \mathrm{m}$ VCSEL. The total thickness of the heterostructure is $0.6 \mu \mathrm{m}$. Mesas, varying in diameter from $4-20 \mu \mathrm{m}$ and $2.5 \mu \mathrm{m}$ in height were formed using a saturated bromine water wet etch. The patterned wafer is cleaned and inserted in the MBE growth chamber for regrowth. A DBR mirror consisting of 10.5 periods of $(1186 \AA) \mathrm{GaAs} /(1338 \AA) \mathrm{AlAs}$ 


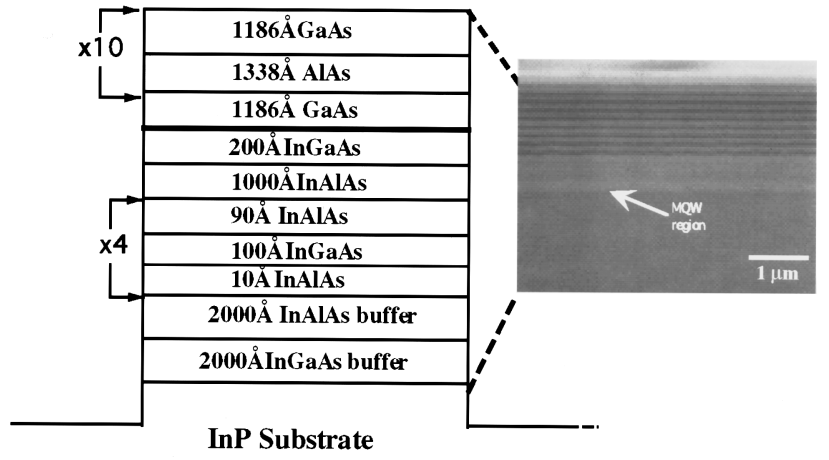

(a)

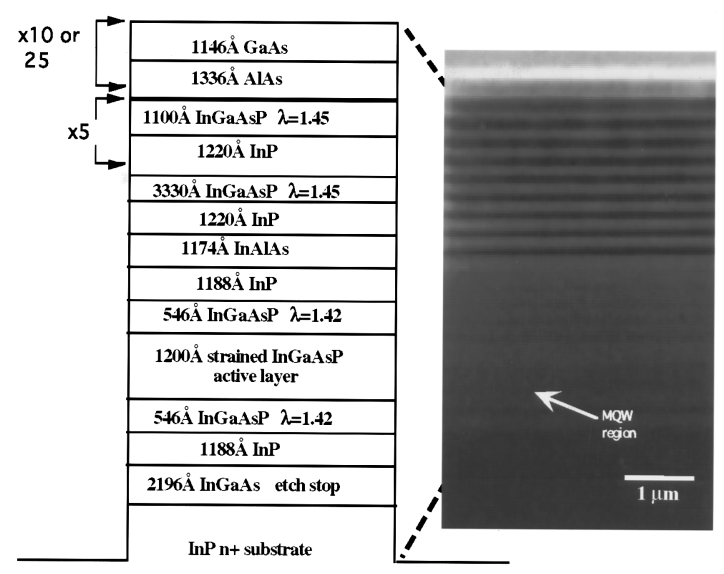

(b)

FIG. 1. Schematic cross sections of regrown GaAs/AlAs Bragg mirrors on (a) InGaAs/InAlAs MQW heterostructure, and (b) InP/InGaAsP MQW VCSEL heterostructure patterned into mesas of sizes ranging 10-40 $\mu \mathrm{m}$. SEM of the first regrown sample is also shown alongside.

layers, designed for $1.6 \mu \mathrm{m}$ wavelength, is grown at a substrate temperature of $500^{\circ} \mathrm{C}$. The total thickness is $2.7 \mu \mathrm{m}$. Also shown in the inset of Fig. 1(a) is the scanning electron micrograph (SEM) of the regrowth DBR on the patterned mesa heterostructure. It may be remembered that the equilibrium critical thickness, according to Matthews and Blakeslee, ${ }^{12}$ for GaAs on InP is less than $100 \AA$. The second MQW structure (structure 2), grown by metalorganic vapor phase epitaxy, at $650^{\circ} \mathrm{C}$, is a $1.55 \mu \mathrm{m}$ VCSEL without semiconductor bottom mirrors. Again, mesas of $15-40 \mu \mathrm{m}$ in diameter and $7 \mu \mathrm{m}$ in height are delineated and 25 periods of $(1146 \AA) \mathrm{GaAs} /(1336 \AA) \mathrm{AlAs}$ Bragg mirror are regrown by MBE.

Marked differences are observed in the morphological, optical, and structural characteristics of the complete heterostructures in the mesa and off-mesa regions. The surface morphology of the regrown mirrors is featureless on top of the mesas, while a distinct cross-hatch pattern is seen in the off-mesa regions. The calculated and measured DBR reflectivities of the 25 periods Bragg mirror on the VCSEL heterostructure (structure 2) are shown in Fig. 2. The measurement was done over the largest mesa. The agreement is good.

In making VCSELs, it is imperative to ascertain that mismatch dislocations at the GaAs-InGaAs layer, if generated, do not propagate downward and deteriorate the optical

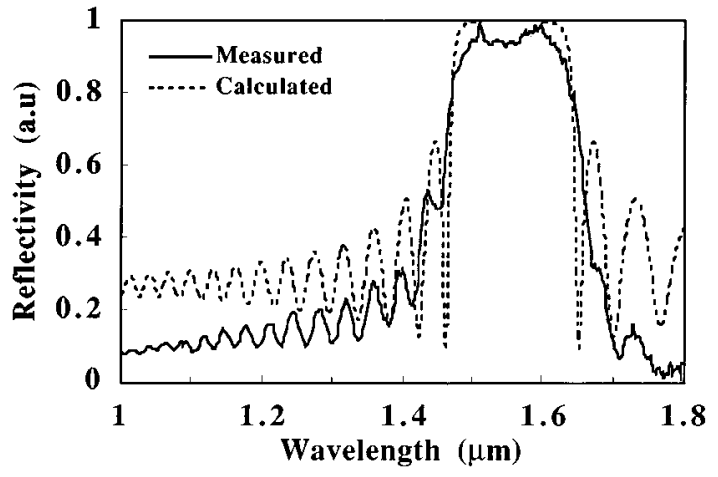

FIG. 2. Measured reflectivity of 25 period GaAs/AlAs Bragg mirror for $\lambda_{p}=1.55 \mu \mathrm{m}$ grown on structure 2 . The calculated reflectivity is also shown for comparison.

properties of the active region. Low temperature $(18 \mathrm{~K})$ photoluminescence (PL) of the InGaAs/InAlAs quantum wells (structure 1) in the different regions and in the different stages of growth and processing were measured with a $\mathrm{He}-\mathrm{Ne}(6328 \AA)$ laser, $1 \mathrm{~m}$ spectrometer, a liquid nitrogen cooled Ge detector, and lock-in amplification. The low temperature PL of the as-grown quantum well is characterized by a single bound exciton peak at $1.45 \mu \mathrm{m}$ with a linewidth (full width at half-maximum) of $16 \mathrm{meV}$. Following regrowth, PL data from the sample show a strong boundexciton related peak originating from the bulk GaAs material in the DBR on top of a $20 \mu \mathrm{m}$ mesa which indicates that the highly mismatched mirrors are of excellent optical quality. Partial removal of the mirror layers by reactive ion etching enabled PL measurement of the MQW region. Shown in Figs. 3(a) and 3(b) are the PL spectra from the top of mesas of size $20 \mu \mathrm{m}$ and $15 \mu \mathrm{m}$, respectively. Photoluminescence data from the same mesa prior to regrowth are superimposed
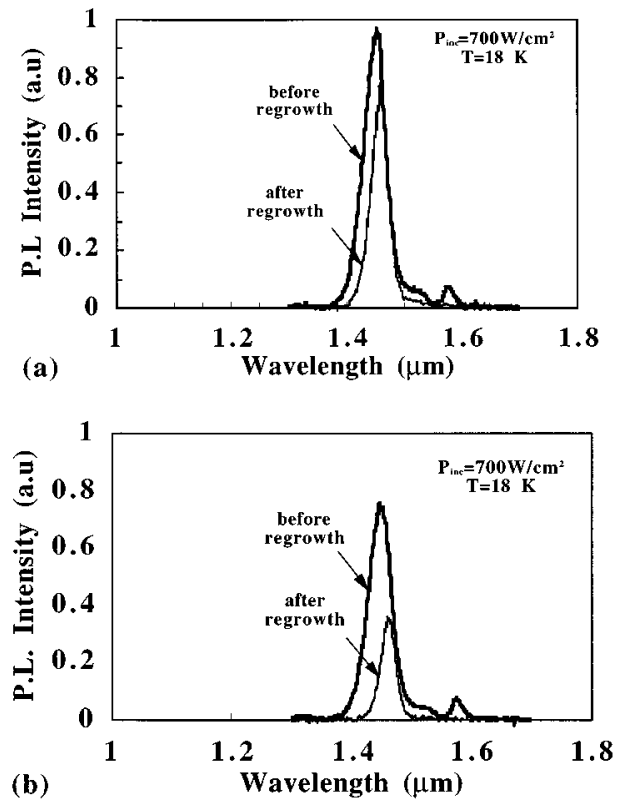

FIG. 3. Low temperature photoluminescence spectra of InGaAs/InAlAs MQW (structure 1) after patterning of mesa compared with spectra after regrowth of 10 periods of GaAs/AlAs DBR for (a) $20 \mu \mathrm{m}$ diam mesa and (b) $15 \mu \mathrm{m}$ diam mesa. The DBRs were partially removed by etching after regrowth to observe the luminescence. 
(a)

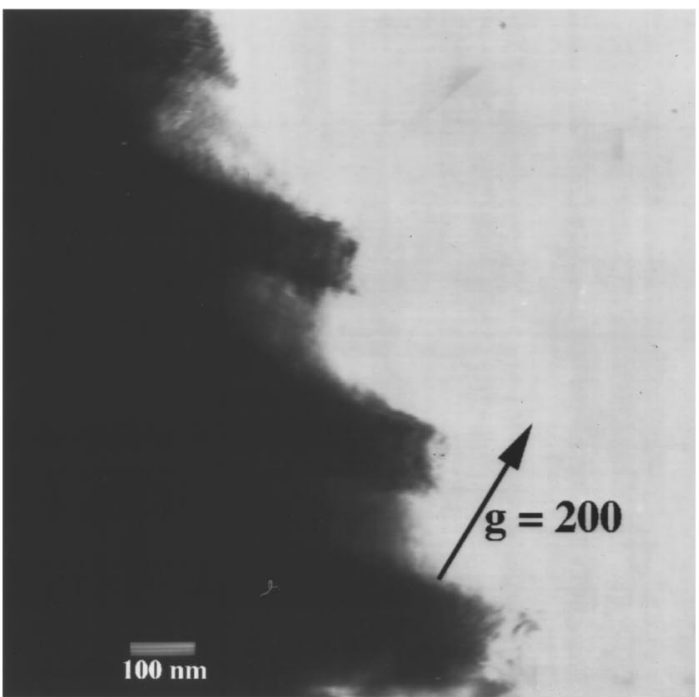

(b)

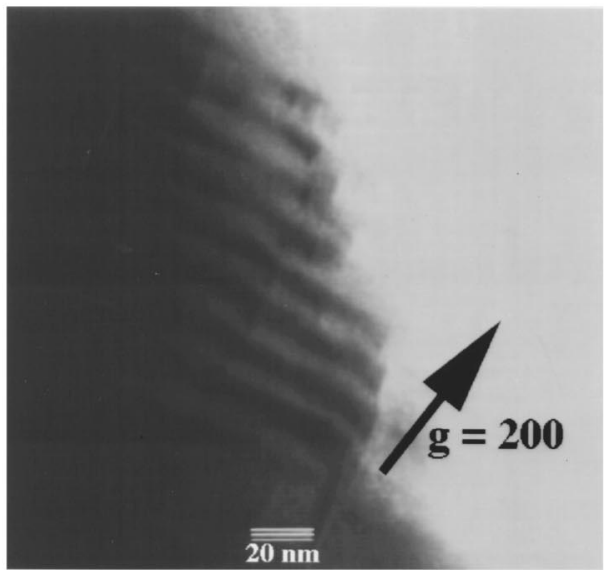

(c)

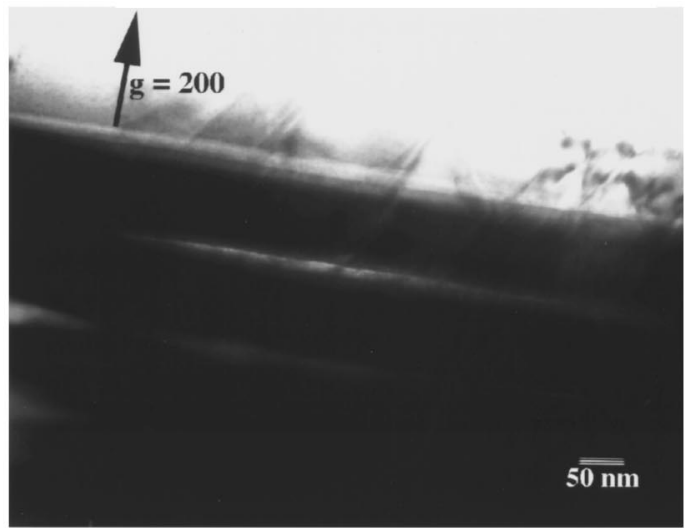

FIG. 4. Cross-sectional transmission electron microscopy micrograph of different regions, after regrowth of GaAs/AlAs DBR: (a) DBR directly above MQW in VCSEL structure; (b) quantum well region in VCSEL structure; and (c) regrown DBR on "infinite" InP substrate.

for comparison. The PL peak position remains unchanged. A slight decrease in peak intensity for the etched mesa before regrowth, compared to the as-grown sample, is due to the relative PL excitation spot size $(30 \mu \mathrm{m})$ becoming larger than the mesa size. Additional decrease in PL intensity after regrowth is observed due to absorption of excitation light in the remaining (unetched) GaAs-based mirror layers. The PL linewidth actually decreases with the reduction in mesa size. These characteristics strongly indicate that VCSELs can be fabricated with the regrown heterostructure.

Finally cross-sectional transmission electron microscopy (XTEM), using a JEOL 2000FX microscope, was done on a $40 \mu \mathrm{m}$ diam mesa VCSEL (structure 2) sample to examine the possible propagation of defects in the InGaAsP quantum wells and the GaAs/AlAs mirror region. The objective was to study these in a patterned region, for which sample preparation is extremely difficult. Bright-field XTEM images are shown in Figs. 4(a) and 4(b). The two most encouraging features are that the MQW region as well as the DBR region directly above it, are free of defects. In comparison, a XTEM image of the regrown 10 periods DBR on unpatterned structure 1 (InP substrate), shows the generation and propagation of dislocations [Fig. 4(c)], as expected.

It is evident that growth of GaAs/AlAs Bragg mirrors on patterned $1.55 \mu \mathrm{m}$ VCSEL structures is feasible. This fabrication technique will allow use of $\mathrm{GaAs} / \mathrm{Al}_{x} \mathrm{O}_{y}$ mirrors, made by selective wet oxidation of the AlAs layers. From the practical point of view one might wonder about the lower Bragg mirror. This can be made with dielectric multilayers, a combination of metal and dielectric multilayers, or with InP/ InGaAsP multilayers. ${ }^{4,13}$ This work is in progress.

In conclusion, it is demonstrated that high quality GaAs/ AlAs Bragg mirrors can be grown directly on small mesas patterned on a $1.55 \mu \mathrm{m}$ InP-based VCSEL structure without generating a large defect density and without deterioration of the active region.

The authors wish to thank R. Jambunathan for useful discussions. This work is being supported by ONR under Grant No. N0014-96-1-0024 and ARO (URI Program) under Grant No. DAAL03-92-G-0109.

${ }^{1}$ J. M. Dallesasse, N. Holonyak, Jr., A. R. Sugg, T. A. Richard, and N. El-Zein, Appl. Phys. Lett. 57, 2844 (1990).

${ }^{2}$ D. L. Huffaker, L. A. Graham, H. Deng, and D. G. Deppe, IEEE Photonics Technol. Lett. 8, 974 (1996).

${ }^{3}$ K. D. Choquette, K. L. Lear, R. P. Schneider, Jr., S. P. Kilcoyne, and K. M. Geib, Electron. Lett. 31, 208 (1995).

${ }^{4}$ M. A. Fisher, Y. Z. Huang, A. J. Dann, D. J. Elton, M. J. Harlow, S. D. Perrin, J. Reed, I. Reid, and M. J. Adams, IEEE Photonics Technol. Lett. 7, 608 (1995).

${ }^{5}$ K. Uomi, S. J. Yoo, A. Scherer, R. Bhat, N. C. Andreadakis, C. E. Zah, M. A. Koza, and T. P. Lee, IEEE Photonics Technol. Lett. 6, 317 (1994).

${ }^{6}$ T. Baba, Y. Yogo, K. Suzuki, F. Koyama, and K. Iga, Electron. Lett. 29, 913 (1993).

${ }^{7}$ D. I. Babic, J. J. Dudley, K. Streubel, R. P. Mirin, J. E. Bowers, and E. L. Hu, Appl. Phys. Lett. 66, 1030 (1995).

${ }^{8}$ E. A. Fitzgerald, G. P. Watson, R. E. Proano, D. G. Ast, J. Appl. Phys. 65, 2220 (1989).

${ }^{9}$ S. V. Ghaisas and A. Madhukar, J. Vac. Sci. Technol. B 7, 264 (1989).

${ }^{10}$ W. Hagen and H. Strunk, Appl. Phys. Lett. 17, 85 (1978).

${ }^{11}$ W. Q. Li, P. K. Bhattacharya, and R. L. Tober, Appl. Phys. Lett. 58, 1931 (1991).

${ }^{12}$ J. W. Matthews and A. E. Blakeslee, J. Cryst. Growth 27, 118 (1974).

${ }^{13}$ C. L. Chua, Z. H. Zhu, and Y. H. Lo, IEEE Photonics Technol. Lett. 7, 444 (1995). 